\title{
Secrecy Outage Analysis for Satellite-Terrestrial Downlink Transmissions
}

\author{
Yongqiang Zhang, Student Member, IEEE, Jia Ye, Student Member, IEEE, Gaofeng Pan, \\ Senior Member, IEEE, and Mohamed-Slim Alouini, Fellow, IEEE
}

\begin{abstract}
This letter investigates the secrecy outage performance of satellite-to-terrestrial downlink transmissions consisting of one legitimate receiver $(D)$ and one eavesdropper $(E)$. To reflect the practical application scenarios, it is assumed that $D$ and $E$ are randomly distributed in the footprint of the satellite. Furthermore, in the considered downlink scenario, $D$ and $E$ are equipped with multiple antennas, and maximal ratio combining (MRC) technique is adopted at both of them to seek the maximum receiving diversity gain. To reveal the impacts of the randomness of the positions of $D$ and $E$, and MRC scheme on the secrecy outage performance of the considered satellite-terrestrial system, the exact and asymptotic closed-form analytical expressions for secrecy outage probability are derived by using stochastic geometry method. Finally, simulation results are given to confirm the accuracy of our proposed analytical models.
\end{abstract}

Index Terms-Maximal ratio combining, satellite-terrestrial communication, secrecy outage probability, stochastic geometry.

\section{INTRODUCTION}

As one of the targets for the future generation of wireless communications is to improve the coverage, to this end, an intuitive solution is to deploy more terrestrial communication networks [1]. However, the deployments of terrestrial communication networks in underserved areas (e.g., rural areas, remote areas, and underpopulated areas) are limited in terms of economical and/or operational feasibility [2]. Seeing as a promising paradigm to cover wider areas with a low cost, satellite-terrestrial communication (STC) system can break such obstacles [3].

On the other hand, with the increasing requirements on secure transmissions, physical-layer (PHY) security has attracted more and more attentions [4], [5]. Recently, there are several existing works on the PHY security of satellite-terrestrial communication systems. In [6], Zheng et al. first investigated the PHY security of a multi-beam satellite system, and then designed an optimal beamforming solution to minimize the total transmit power under users' secrecy rate constraints. Bankey et al. studied the PHY security of a downlink hybrid satellite-terrestrial multi-relay network in [7]. The idea of exploiting the terrestrial resources to enhance PHY of satellite transmission has been investigated in [8]. The authors of [9] considered a practical unknown eavesdropper scenario and presented a low-complexity PHY scheme. Moreover, the secrecy performance of a hybrid satellite and free-space optical communication system was investigated in [10]. The authors

Manuscript received Jan. 22, 2020; revised Apr. 30, 2020; accepted May 30,2020 . This work was supported by a grant from the office of sponsored research at KAUST. The associate editor coordinating the review of this paper and approving it for publication was Yongming Huang. (Corresponding author: Gaofeng Pan.)

The authors are with Computer, Electrical and Mathematical Sciences and Engineering Division, King Abdullah University of Science and Technology (KAUST), Thuwal 23955-6900, Saudi Arabia. (e-mail: yongqiang.zhang.2,jia.ye,gaofeng.pan,slim.alouini@kaust.edu.sa )

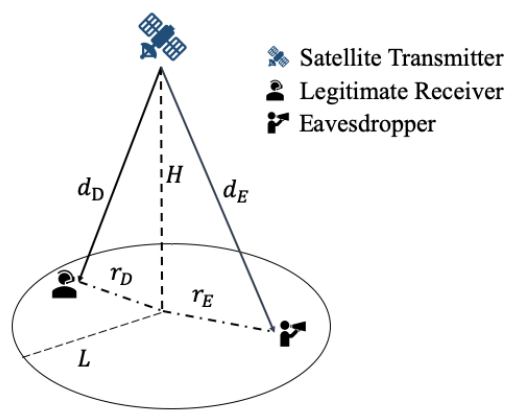

Fig. 1: System model.

of [11] addressed a secrecy rate maximization problems for a heterogeneous satellite-terrestrial communication system. In [12], Li et al. analyzed the secrecy performance of a land mobile satellite system with one legitimate receiver and multiple single-antenna eavesdroppers. Further, the secrecy performance of satellite communication systems with a multiantenna legitimate receiver and multiple single-antenna eavesdroppers was evaluated in [13]

However, there are few works about the PHY security of STC system while considering the randomness of the positions of the terminals, especially combined with multiantenna diversity, which motivates us for this work.

In this letter, making use of stochastic geometry theory, we study the secrecy performance of an STC scenario including one legitimate receiver and one eavesdropper, while considering the randomness of the locations of legitimate and eavesdropping receivers. We assume that both legitimate and eavesdropping receivers are equipped with multiple antennas. Since maximal ratio combining (MRC) scheme outperforms selection combining (SC) scheme [14] because all copies of the received signals from each antenna are utilized, we consider all terrestrial receivers employ MRC scheme to achieve the maximal receiving diversity gain to realize the best performance of themselves'. The main contributions of this letter can be summarized as follows:

1) The statistical characteristics of the received signal-tonoise ratio (SNR) at terrestrial receivers, namely, probability density function (PDF) and cumulative distribution function (CDF), are derived while considering the impact of the randomness of the distributions of terrestrial receivers;

2) We derive the exact and asymptotic closed-form analytical expressions for secrecy outage probability (SOP) to uncover the influences of the randomness of the locations of all terrestrial receivers and MRC scheme.

\section{SySTEM MOdEL}

As shown in Fig. 1, in this work, we consider a satelliteterrestrial transmission which consists of one satellite $(S)$, one 
legitimate terrestrial receiver $(D)$, and one terrestrial eavesdroppers $(E)$. For the convenience of subsequent derivations, we treat the coverage area of the satellite as a circle with radius $L$. The projection of the satellite on the ground is the origin of this circle. We assume that $D$ and $E$ are equipped with $N_{D} \geq 2$ and $N_{E} \geq 2$ antennas, respectively.

Moreover, the locations of the two terrestrial receivers are modeled as a uniform distribution. According to [15], we can calculate the distance from the terrestrial receivers to the projection of $S$ by $\mathcal{M}$, the PDF of which can be written as $f_{\mathcal{M}}(x)=\frac{1}{\pi L^{2}}$.

Shadowed-Rician model proposed in [16] is adopted in this work to capture the statistical property of the satelliteterrestrial link. Then, the PDF of the channel gain for the link from $S$ to the $i$ th antenna at receiver $j$ is given by

$$
\begin{aligned}
f_{\left|h_{j i}\right|^{2}}(x)= & \left(\frac{2 m_{j} b_{j}}{2 m_{j} b_{j}+\Omega_{j}}\right)^{m_{j}} \frac{1}{2 b_{j}} \exp \left(-\frac{x}{2 b_{j}}\right) \\
& \cdot{ }_{1} F_{1}\left(m_{j}, 1, \frac{\Omega_{j} x}{2 b_{j}\left(2 m_{j} b_{j}+\Omega_{j}\right)}\right),
\end{aligned}
$$

where $j \in\{D, E\}, i \in\left\{1, \ldots, N_{j}\right\}, 2 b_{j}$ and $\Omega_{j}$ are the average power of scatter and line-of-sight components, respectively, $m_{j}$ denotes the fading severity parameter, ${ }_{1} F_{1}(\cdot ; \cdot ; \cdot)$ represents the first kind confluent hypergeometric function.

Further, we can derive the SNR of the $i$ th antenna at receiver $j$ as $\gamma_{j i}=\frac{P_{s}\left|h_{j i}\right|^{2}}{\sigma^{2} d_{j}^{n}}$, where $P_{s}$ denotes the transmit power at $S^{1}$, $\sigma^{2}$ represents the average power of the additive white Gaussian noise (AWGN), $n$ is the path-loss factor for the link from $S$ to terrestrial receiver $j, d_{j}=\sqrt{H^{2}+r_{j}^{2}}$ is the distance between $S$ and terrestrial receiver $j, r_{j}$ denotes the distance from the receiver $j$ to the origin of the terrestrial disk, and $H$ denotes the height of the satellite.

Let $\lambda_{j i}=\frac{P_{s}\left|h_{j i}\right|^{2}}{\sigma^{2}}\left(0 \leq j \leq N_{j}\right)$, the PDF and CDF of $\lambda_{j i}$ can be given by

$$
f_{\lambda_{j i}}(x)=\alpha_{j} \sum_{k=0}^{m_{j}-1} \frac{\varsigma_{j}(k)}{\bar{\lambda}^{k+1}} x^{k} \exp \left(-\frac{\beta_{j}-\delta_{j}}{\bar{\lambda}} x\right)
$$

and

$$
\begin{aligned}
F_{\lambda_{j i}}(x)=1 & -\alpha_{j} \sum_{k=0}^{m_{j}-1} \frac{\varsigma_{j}(k)}{\bar{\lambda}^{k+1}} \sum_{p=0}^{k} \frac{k !}{p !}\left(\frac{\beta_{j}-\delta_{j}}{\bar{\lambda}}\right)^{-(k+1-p)} \\
& \cdot x^{p} \exp \left(-\frac{\beta_{j}-\delta_{j}}{\bar{\lambda}} x\right)
\end{aligned}
$$

respectively, where $\bar{\lambda}=\frac{P_{\mathrm{S}}}{\sigma^{2}}, \varsigma_{j}(k)=\frac{(-1)^{k}\left(1-m_{j}\right)_{k} \delta_{j}^{k}}{(k !)^{2}}$, $\alpha_{j}=\left(\frac{2_{j} b m_{j}}{2 b_{j} m_{j}+\Omega_{j}}\right)^{m_{j}} /\left(2 b_{j}\right), \quad \beta_{j}=\frac{1}{2 b_{j}}, \quad \delta_{j}=\frac{\Omega_{j}}{2 b_{j}\left(2 b_{j} m_{j}+\Omega_{j}\right)}$, and $(t)_{k}=t(t+1) \cdots(t+k-1)$ is the Pochhammer symbol [17].

Using the PDF of $\mathcal{M}$, we can derive the CDF and PDF of $r_{j}$ as $F_{r_{j}}(x)=\int_{0}^{2 \pi} \int_{0}^{x} \frac{r}{\pi L^{2}} \mathrm{~d} r \mathrm{~d} \theta=\frac{x^{2}}{L^{2}}$ and $f_{r_{j}}(x)=$ $\frac{2 x}{L^{2}}, \quad 0 \leq x \leq L$, respectively. Further, the PDF of $d_{j}^{n}$ can

\footnotetext{
${ }^{1} \mathrm{Here}$, it is also assumed that there is no channel state information at the satellite, which meaning that the transmit power at the satellite will not be adjusted during the information transmission process. Then, secrecy outage events will happen.
}

be given as $f_{d_{j}^{n}}(x)=\frac{2 x^{\frac{2}{n}-1}}{n L^{2}}$. By using MRC technique, the received SNR at receiver $j$ can be expressed as $\gamma_{j}=\sum_{i=0}^{N_{j}} \gamma_{j i}=$ $\left(\sum_{i=0}^{N_{j}} \lambda_{j i}\right) / d_{j}^{n}$

\section{Secrecy Outage AnAlysis}

As suggested by [18], the PDF of $\lambda_{j}=\sum_{i=0}^{N_{j}} \lambda_{j i}$ is given by

$$
\begin{aligned}
f_{\lambda_{j}}(x)= & \left(\frac{2 b_{j} m_{j}}{\Omega_{j}+2 b_{j} m_{j}}\right)^{\tilde{m}_{j}} \cdot \frac{x^{N-1} \exp \left(-\frac{x}{2 b_{j} \bar{\lambda}}\right)}{\Gamma\left(N_{j}\right)\left(2 b_{j}\right)^{N_{j}} \bar{\lambda}^{N}} \\
& \cdot{ }_{1} F_{1}\left(\tilde{m}_{j}, N_{j} ; \frac{\Omega_{j} x}{\bar{\lambda} 2 b_{j}\left(2 b_{j} m_{j}+\Omega_{j}\right)}\right)
\end{aligned}
$$

where $\bar{\lambda}=\frac{P_{\mathrm{S}}}{\sigma^{2}}$ and $\tilde{m}_{j}=\sum_{i=0}^{N_{j}} m_{j}$.

By using Kummers transform method to represent the hypergeometric function [19], we can rewrite (4) as

$$
f_{\lambda_{j}}(x)=\frac{\tilde{\alpha}_{j}}{\tilde{\beta}_{j}} \sum_{k=0}^{\tilde{m}_{j}-N_{j}} \theta_{j k} \exp \left(-\psi_{j} x\right) x^{k+N_{j}-1},
$$

where $\tilde{\alpha}_{j}=\left(\frac{2 b_{j} \tilde{m}_{j}}{\tilde{\Omega}_{j}+2 b_{j} \tilde{m}_{j}}\right)^{\tilde{m}_{j}}, \tilde{\beta}_{j}=\Gamma\left(N_{j}\right)\left(2 b_{j}\right)^{N_{j}} \bar{\lambda}^{N_{j}}, \tilde{\sigma}_{j}=$ $\frac{\Omega_{j}}{\bar{\lambda} 2 b_{j}\left(2 b_{j} m_{j}+\Omega_{j}\right)}, \theta_{j k}=\frac{\left(\tilde{m}_{j}-N\right) ! \sigma_{j}^{k}}{k !\left(\tilde{m}_{j}-N-k\right) !(N)_{k}}$, and $\psi_{j}=\frac{1}{2 b_{j} \lambda}-\sigma_{j}$. Then, the CDF of $\lambda_{j}=\sum_{i=0}^{N_{j}} \lambda_{j i}$ can be written as

$$
\begin{gathered}
F_{\lambda_{j}}(x)=1-\frac{\tilde{\alpha}_{j}}{\tilde{\beta}_{j}} \sum_{k=0}^{\tilde{m}_{j}-N_{j}} \theta_{j k} \sum_{p=0}^{k+N_{j}-1} \tilde{\varsigma}_{k p}\left(\psi_{j}\right)^{-\left(k+N_{j}-p\right)} \\
\cdot x^{p} \exp \left(-\psi_{j} x\right)
\end{gathered}
$$

where $\tilde{\varsigma}_{k p}=\left(k+N_{j}-1\right) ! / p !$.

The instantaneous secrecy capacity of the considered system can be expressed as

$$
C_{s}\left(\gamma_{D}, \gamma_{E}\right)=\log _{2}\left(1+\gamma_{D}\right)-\log _{2}\left(1+\gamma_{E}\right) \text {. }
$$

Then, the outage is defined as the event that the eavesdropping links secrecy capacity is negative. Therefore, the SOP of the considered system is given as

$$
P_{S O P}=\operatorname{Pr}\left\{C_{s}\left(\gamma_{D}, \gamma_{E}\right) \leq 0\right\}
$$

Corollary 1. A closed-form analytical expression for SOP without considering the the randomness of the positions of $D$ and $E$ is given by

$$
\begin{aligned}
P_{S O P \mid d_{D}, d_{E}}=1- & \sum_{k=0}^{\tilde{m}_{D}-N_{D}} \sum_{p=0}^{k+N_{D}-1} \sum_{l=0}^{\tilde{m}_{E}-N_{E}} \Xi_{k, p, l}\left(\frac{d_{D}^{n}}{d_{E}^{n}}\right)^{p} \\
& \cdot \frac{\Gamma(l+p+N)}{\left(\psi_{D} \frac{d_{D}^{n}}{d_{E}^{n}}+\psi_{E}\right)^{l+p+N}} .
\end{aligned}
$$


Proof:

$$
\begin{aligned}
P_{S O P \mid d_{D}, d_{E}}= & \operatorname{Pr}\left\{\gamma_{D} \leq \gamma_{E} \mid d_{D}, d_{E}\right\} \\
= & \operatorname{Pr}\left\{\lambda_{D} \leq \frac{\lambda_{E} d_{D}^{n}}{d_{E}^{n}} \mid d_{D}, d_{E}\right\} \\
=1 & -\int_{0}^{\infty} \sum_{k=0}^{\tilde{m}_{D}-N_{D}} \sum_{p=0}^{k+N_{D}-1} \sum_{l=0}^{\tilde{m}_{E}-N_{E}} \Xi_{k, p, l}\left(\frac{d_{D}^{n}}{d_{E}^{n}}\right)^{p} \\
& \cdot \exp \left[-\left(\psi_{D} \frac{d_{D}^{n}}{d_{E}^{n}}+\psi_{E}\right) x\right] x^{l+p+N_{E}-1} \mathrm{~d} x,
\end{aligned}
$$

where $\Xi_{k, p, l}=\tilde{\alpha}_{D} \tilde{\alpha}_{E} \theta_{j k} \theta_{E l} \tilde{\zeta}_{k p} /\left(\tilde{\beta}_{D} \tilde{\beta}_{E} \psi_{D}^{k+N_{D}-p}\right)$. In the following, we use $\sum_{k, p, l}$ instead of $\sum_{k=0}^{\tilde{m}_{D}-N_{D}} \sum_{p=0}^{k+N_{D}-1} \sum_{l=0}^{\tilde{m}_{E}-N_{E}}$ for simplification.

Let $I$ denote the integral part in (10), and using [17, Eq. (3.326.2.10)], we have

$$
I=\sum_{k, p, l} \Xi_{k, p, l}\left(\frac{d_{D}^{n}}{d_{E}^{n}}\right)^{p} \frac{\Gamma(l+p+N)}{\left(\psi_{D} \frac{d_{D}^{n}}{d_{E}^{n}}+\psi_{E}\right)^{l+p+N}} .
$$

This proof is concluded by substituting (11) into (10).

Taking the randomness of $d_{D}$ and $d_{E}$ into account, and using Corollary 1, $S O P$ can be written as

$$
\begin{aligned}
S O P= & \int_{d_{\min }^{n}}^{d_{\max }^{n}} \int_{d_{\min }^{n}}^{d_{\max }^{n}} \frac{2 x_{0}^{\frac{2}{n}-1}}{n L^{2}} \frac{2 x_{e}^{\frac{2}{n}-1}}{n L^{2}}[1- \\
& \left.\sum_{k, p, l} \Xi_{k, p, l}\left(\frac{x_{0}}{x_{e}}\right)^{p} \frac{\Gamma(l+p+N)}{\left(\psi_{D} \frac{x_{0}}{x_{e}}+\psi_{E}\right)^{l+p+N}}\right] \mathrm{d} x_{0} \mathrm{~d} x_{e} \\
= & 1-\sum_{k, p, l} \Xi_{k, p, l} \frac{4 \Gamma(l+p+N)}{n^{2} L^{4}} \int_{d_{\min }^{n}}^{d_{\max }^{n}} x_{e}^{l+N+\frac{2}{n}-1} \\
& \int_{d_{\min }^{n}}^{d_{\max }^{n}} \frac{x_{0}{ }^{\frac{2}{n}+p-1}}{\left(\psi_{D} x_{0}+\psi_{E} x_{e}\right)^{l+p+N}} \mathrm{~d} x_{0} \mathrm{~d} x_{e},
\end{aligned}
$$

where $d_{\max }=\sqrt{L^{2}+H^{2}}$ and $d_{\min }=H$, respectively.

Corollary 2. A closed-form analytical expression for SOP is given in (13), as shown on the top of this page.

Proof: By using [20, Eq. (1.2.4.4)], we can obtain the closed form of the inner integral in (12) as

$$
\begin{aligned}
I_{1} & =\int_{d_{\min }^{n}}^{d_{\max }^{n}} \frac{x_{0}^{\frac{2}{n}+p-1}}{\left(\psi_{D} x_{0}+\psi_{E} x_{e}\right)^{l+p+N}} \mathrm{~d} x_{0} \\
& =\frac{\left(d_{\min }^{n}\right)^{-\mu_{l}}}{\mu_{l} \psi_{D}{ }^{\nu_{p l}}} \cdot{ }_{2} F_{1}\left(\nu_{p l}, \mu_{l} ; 1+\mu_{l},-\frac{\psi_{E} x_{e}}{\psi_{D} d_{\min }^{n}}\right) \\
& -\frac{\left(d_{\max }^{n}\right)^{-\mu_{l}}}{\mu_{l} \psi_{D}{ }^{\nu_{p l}}} \cdot{ }_{2} F_{1}\left(\nu_{p l}, \mu_{l} ; 1+\mu_{l},-\frac{\psi_{E} x_{e}}{\psi_{D} d_{\max }^{n}}\right),
\end{aligned}
$$

where $\nu_{p l}=l+p+N, \mu_{l}=N+l-2 / n$.

By employing (15), we can deuce (12) into (14), as shown on the top of this page, where ${ }_{2} F_{1}(\cdot, \cdot ; \cdot, \cdot)$ is the hypergeometric function.

To simplify the analysis, we use Meijers G-function to represent the hypergeometric function and define a new function $g(\tau, \xi, l, p)$ as

$$
\begin{aligned}
g(\tau, \xi, l, p)= & \int_{0}^{\tau} x^{\eta_{l}}{ }_{2} F_{1}\left(\nu_{p l}, \mu_{l} ; 1+\mu_{l},-\xi x\right) \mathrm{d} x \\
= & \int_{0}^{\xi \tau}\left(\frac{1}{\xi}\right)^{\eta_{l}+1} t^{\eta_{l}}{ }_{2} F_{1}\left(\nu_{p l}, \mu_{l} ; 1+\mu_{l},-t\right) \mathrm{d} t \\
= & \int_{0}^{\xi \tau}\left(\frac{1}{\xi}\right)^{\eta_{l}+1} \frac{\Gamma\left(1+\mu_{l}\right)}{\Gamma\left(\nu_{p l}\right) \Gamma\left(\mu_{l}\right)} t^{\eta_{l}} \\
& \cdot \mathrm{G}_{22}^{12}\left[\begin{array}{c}
1-\nu_{p l}, 1-\mu_{l} \\
0,-\mu_{l}
\end{array}\right] \mathrm{d} t
\end{aligned}
$$

where $\eta_{l}=l+N+2 / n-1$, and $\mathrm{G}_{p, q}^{m, n}[\cdot \mid \cdot]$ denotes the single variable Meijers G-function.

Using [21, Eq. (26)], we can rewrite (16) as

$$
\begin{aligned}
g(\tau, \xi, l, p)= & \left(\frac{1}{\xi}\right)^{\eta_{l}+1} \frac{\Gamma\left(1+\mu_{l}\right)}{\Gamma\left(\nu_{p l}\right) \Gamma\left(\mu_{l}\right)}(\xi \tau)^{\eta_{l}+1} \\
& \cdot \mathrm{G}_{33}^{13}\left[\begin{array}{c|c}
\xi \tau \mid \nu_{p l}, 1-\mu_{l},-\eta_{l} \\
0,-1-\eta_{l},-\mu_{l}
\end{array}\right] .
\end{aligned}
$$
(10).

This proof is concluded by substituting (17) and (15) into

\section{Asymptotic Analysis}

Observing the complexity of (13) that hinders it from being applied into practical applications, in this section the asymptotic outage behaviors in high SNR region will be investigated, which can serve as a useful reference in the engineering designs of the considered systems.

By employing [17, Eq. (3.351.1)], the CDF of $\lambda_{j}$ can be further expressed as

$$
F_{\lambda_{j}}(x)=\frac{\tilde{\alpha}_{j}}{\tilde{\beta}_{j}} \sum_{k=0}^{\tilde{m}_{j}-N_{j}} \theta_{j k} \frac{\gamma\left(k+N_{j}, \psi_{j} x\right)}{\psi_{j}{ }^{k+N_{j}}},
$$

where $\gamma(\cdot, \cdot)$ denotes the lower incomplete gamma function.

In high SNR region (i.e., $\psi_{j} \rightarrow 0$ ), using the series representation of $\gamma(\cdot, \cdot)$ [17, Eq. (8.354.1)], we can derive the aympotic CDF of $\lambda_{j}$ as

$$
F_{\lambda_{j}}^{\infty}(x)=\frac{\tilde{\alpha}_{j}}{\tilde{\beta}_{j}} \sum_{k=0}^{\tilde{m}_{j}-N_{j}} \theta_{j k} \frac{\left(\psi_{j} x\right)^{k+N_{j}}}{\left(k+N_{j}\right) \psi_{j}^{k+N_{j}}} .
$$

Corollary 3. The closed-form analytical expression for the asymptotic $S O P(A S O P)$ is given by

$$
\begin{gathered}
P_{S O P}^{\infty}=\frac{4}{n^{2} L^{4}} \sum_{k=0}^{\tilde{m}_{D}-N_{D}} \sum_{l=0}^{\tilde{m}_{E}-N_{E}} o_{k l}\left(\frac{d_{\max }^{n\left(k+N_{D}\right)+2}}{2 / n+k+N_{D}}\right. \\
\left.-\frac{d_{\min }^{n\left(k+N_{D}\right)+2}}{2 / n+k+N_{D}}\right)\left(\frac{d_{\max }^{2-n\left(k-N_{D}\right)}}{2 / n-k-N_{D}}-\frac{d_{\min }^{2-n\left(k-N_{D}\right)}}{2 / n-k-N_{D}}\right),
\end{gathered}
$$

where $o_{k l}=\frac{\tilde{\alpha}_{D} \tilde{\alpha}_{E} \theta_{D k} \theta_{E k} \Gamma\left(k+l+N_{D}+N_{E}\right)}{\tilde{\beta}_{D} \tilde{\beta}_{E}\left(k+N_{D}\right) \psi_{E}{ }^{2 k+N_{D}+N_{E}}}$.

Proof: Similar to the derivation of $P_{S O P}$, we will first calculate $P_{S O P \mid d_{D}, d_{E}}^{\infty}$. 


$$
\begin{aligned}
S O P= & 1-\sum_{k, p, l} \Xi_{k, p, l} \frac{4 \Gamma(l+p+N)}{n^{2} L^{4}}\left\{\frac{\left(d_{\min }^{n}\right)^{-\mu_{l}}}{\mu_{l} \psi_{D} \nu^{\nu l}}\left[g\left(d_{\max }^{n}, \frac{\psi_{E}}{\psi_{0} d_{\min }^{n}}, l, p\right)-g\left(d_{\min }^{n}, \frac{\psi_{E}}{\psi_{D} d_{\min }^{n}}, l, p\right)\right]\right. \\
& \left.-\frac{\left(d_{\max }^{n}\right)^{-\mu_{l}}}{\mu_{l} \psi_{D} \nu_{p l}}\left[g\left(d_{\max }^{n}, \frac{\psi_{E}}{\psi_{D} d_{\max }^{n}}, l, p\right)-g\left(d_{\min }^{n}, \frac{\psi_{E}}{\psi_{D} d_{\max }^{n}}, l, p\right)\right]\right\} .
\end{aligned}
$$

$$
\begin{array}{r}
S O P=1-\sum_{k, p, l} \Xi_{k, p, l} \frac{4 \Gamma(l+p+N)}{n^{2} L^{4}} \int_{d_{\min }^{n}}^{d_{\max }^{n}} x_{e}^{l+N+\frac{2}{n}-1}\left[\frac{\left(d_{\min }^{n}\right)^{-\mu_{l}}}{\mu_{l} \psi_{D} \nu_{p l}} \cdot{ }_{2} F_{1}\left(\nu_{p l}, \mu_{l} ; 1+\mu_{l},-\frac{\psi_{E} x_{e}}{\psi_{D} d_{\min }^{n}}\right)\right. \\
\left.-\frac{\left(d_{\max }^{n}\right)^{-\mu_{l}}}{\mu_{l} \psi_{D} \nu_{p l}} \cdot{ }_{2} F_{1}\left(\nu_{p l}, \mu_{l} ; 1+\mu_{l},-\frac{\psi_{E} x_{e}}{\psi_{D} d_{\max }^{n}}\right)\right] \mathrm{d} x_{e} .
\end{array}
$$

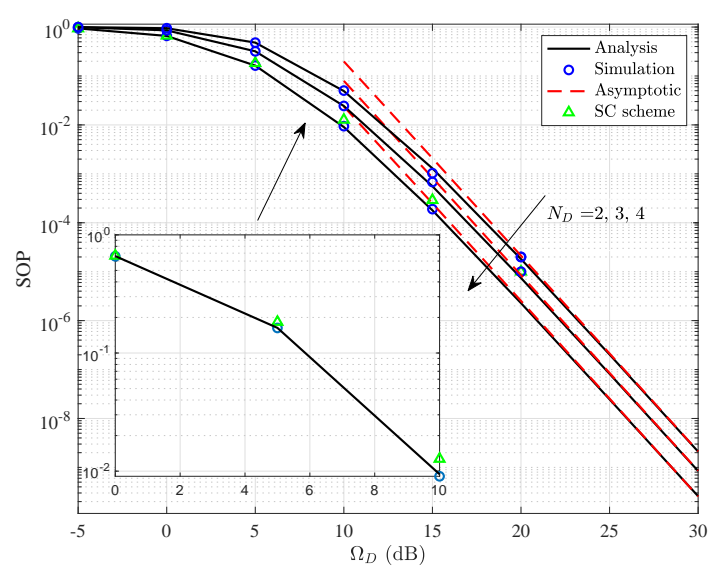

Fig. 2: SOP vs. $\Omega_{D}$ for various $N_{D}$.

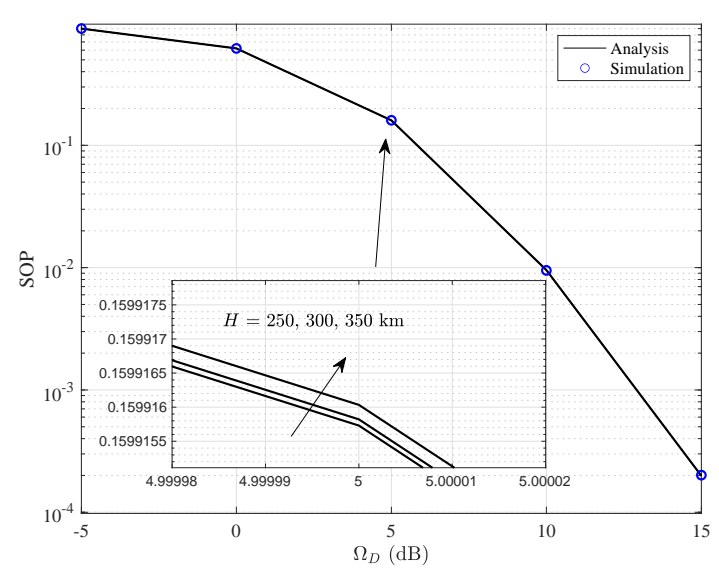

Fig. 3: SOP vs. $\Omega_{D}$ for various $H$.
Making use of [17, Eq. (3.326.2.10)], we can obtain

$$
\begin{aligned}
P_{S O P \mid d_{D}, d_{E}}^{\infty}= & \int_{0}^{\infty} \frac{\tilde{\alpha}_{D}}{\tilde{\beta}_{D}} \frac{\tilde{\alpha}_{E}}{\tilde{\beta}_{E}} \sum_{k=0}^{\tilde{m}_{D}-N_{D}} \sum_{l=0}^{\tilde{m}_{E}-N_{E}} \theta_{D k} \theta_{E k} \\
& \frac{x^{k+l+N_{D}+N_{E}-1}}{k+N_{D}} \exp \left(-\psi_{E} x\right)\left(\frac{d_{D}}{d_{E}}\right)^{k+N_{D}} \mathrm{~d} x \\
= & \sum_{k=0}^{\tilde{m}_{D}-N_{D}} \sum_{l=0}^{\tilde{m}_{E}-N_{E}} o_{k l}\left(\frac{d_{D}}{d_{E}}\right)^{k+N_{D}}
\end{aligned}
$$

Further, considering the randomness of $d_{D}$ and $d_{E}$, we can derive the closed-form analytical expression for ASOP as (20) by calculating a elementary rational function integral.

\section{NuMERiCAL RESUlts}

In this section, we present Monte Carlo simulation to validate our analytical expressions for SOP. In each case, we perform at least $10^{5}$ times of the realizations of the channel and the positions of terrestrial receivers. Besides, we set $n=2$, $m_{E}=m_{D}=2, b_{E}=0.25, b_{D}=0.36, \Omega_{E}=1 \mathrm{~dB}, N_{D}=2$, and $L=15 \mathrm{~km}^{2}$.

Fig. 2 depicts that the changes in SOP versus $\Omega_{D}$. We can observe that SOP decreases when $\Omega_{D}$ improves. This is

\footnotetext{
${ }^{2}$ In this section, we set the height and the coverage size of the footprint of $S$ by considering the case of low earth orbit satellites.
}

reasonable since that high $\Omega_{D}$ leads to improved legitimate channel quality. We can observe that for fixed $\Omega_{D}$, SOP increase with $N_{D}$ increase, as MRC diversity gain increases at the legitimate receiver. In the high $\Omega_{D}$ region, Fig. 2 indicates that the gap between asymptotic and the exact results is negligible. Fig. 2 also shows that the SOP of MRC scheme outperforms the one of SC scheme. Besides, Fig. 3 indicates that the increment of $H$ shows a weak impact on SOP. The differences among the SOP for various $H$ from 250 $\mathrm{km}$ to $350 \mathrm{~km}$ is very narrow (roughly on the orders of $10^{-6}$. Recalling that the expression for the received SNR at terrestrial receivers, adjusting the height of the satellite leads to an approximate identical influence to both legitimate and eavesdropping receivers.

\section{CONCLUSION}

In this letter, we investigated the secrecy outage performance in multi-antenna satellite-terrestrial transmissions by deriving the closed-form expressions for the exact and asymptotic SOP, while considering the randomness of the positions of legitimate and eavesdropping receivers. Numerical results reveal that the height of the satellite exhibits a weak effect on SOP, while the number of antennas at the eavesdropper shows a positive impact on it. As expected, compared with SC scheme, MRC scheme can achieve a better performance in terms of SOP. 


\section{REFERENCES}

[1] O. Y. Kolawole, S. Vuppala, M. Sellathurai, and T. Ratnarajah, "On the performance of cognitive satellite-terrestrial networks," IEEE Trans. Cogn. Commun. Netw., vol. 3, no. 4, pp. 668-683, Dec. 2017.

[2] G. Zheng, S. Chatzinotas, and B. Ottersten, "Generic optimization of linear precoding in multibeam satellite systems," IEEE Trans. Wireless Commun., vol. 11, no. 6, pp. 2308-2320, Jun. 2012.

[3] W. W. Wu, "Satellite communications," Proc. IEEE, vol. 85, no. 6, pp. 998-1010, Jun. 1997.

[4] G. Pan, J. Ye, and Z. Ding, "Secure hybrid VLC-RF systems with light energy harvesting," IEEE Trans. Commun., vol. 65, no. 10, pp. 43484359, Oct. 2017.

[5] J. Ye, C. Zhang, H. Lei, G. Pan, and Z. Ding, "Secure UAV-to-UAV systems with spatially random UAVs," IEEE Wireless Commun. Lett., vol. 8, no. 2, pp. 564-567, Apr. 2019.

[6] G. Zheng, P. Arapoglou, and B. Ottersten, "Physical layer security in multibeam satellite systems," IEEE Trans. Wireless Commun., vol. 11, no. 2, pp. 852-863, Feb. 2012.

[7] V. Bankey and P. K. Upadhyay, "Physical layer security of multiuser multirelay hybrid satellite-terrestrial relay networks," IEEE Trans. Veh. Technol., vol. 68, no. 3, pp. 2488-2501, Mar. 2019.

[8] M. Lin, Z. Lin, W.-P. Zhu, and J.-B. Wang, "Joint beamforming for secure communication in cognitive satellite terrestrial networks," IEEE J. Sel. Areas Commun., vol. 36, no. 5, pp. 1017-1029, May 2018.

[9] Z. Lin, M. Lin, B. Champagne, W.-P. Zhu, and N. Al-Dhahir, "Secure beamforming for cognitive satellite terrestrial networks with unknown eavesdroppers," IEEE Syst. J., pp. 1-4, 2020.

[10] Y. Ai, A. Mathur, M. Cheffena, M. R. Bhatnagar, and H. Lei, "Physical layer security of hybrid satellite-FSO cooperative systems," IEEE IEEE Photon. J., vol. 11, no. 1, pp. 1-14, Feb. 2019.

[11] J. Du, C. Jiang, H. Zhang, X. Wang, Y. Ren, and M. Debbah, "Secure satellite-terrestrial transmission over incumbent terrestrial networks via cooperative beamforming," IEEE J. Sel. Areas Commun., vol. 36, no. 7, pp. 1367-1382, Jul. 2018.

[12] Y. Li, K. An, T. Liang, and X. Yan, "Secrecy performance of land mobile satellite systems with imperfect channel estimation and multiple eavesdroppers," IEEE Access, vol. 7, pp. 31 751-31 761, 2019.

[13] K. Guo, K. An, Y. Huang, and B. Zhang, "Physical layer security of multiuser satellite communication systems with channel estimation error and multiple eavesdroppers," IEEE Access, vol. 7, pp. 96253-96262, Jul. 2019.

[14] H. Zhao, Y. Tan, G. Pan, Y. Chen, and N. Yang, "Secrecy outage on transmit antenna selection/maximal ratio combining in mimo cognitive radio networks," IEEE Trans. Veh. Technol., vol. 65, no. 12, pp. 10236 10242, Dec. 2016.

[15] G. Pan, J. Ye, and Z. Ding, "On secure VLC systems with spatially random terminals," IEEE Commun. Lett., vol. 21, no. 3, pp. 492-495, Mar. 2017.

[16] A. Abdi, W. C. Lau, M.-S. Alouini, and M. Kaveh, "A new simple model for land mobile satellite channels: first and second order statistics," IEEE Trans. Wireless Commun., vol. 2, no. 3, pp. 519-528, May 2003.

[17] I. S. Gradshteyn and I. M. Ryzhik, Table of Integrals, Series, and Products, 7th ed. San Diego: Academic Press, 2007.

[18] Q. Huang, M. Lin, W.-P. Zhu, S. Chatzinotas, and M.-S. Alouini, "Performance analysis of integrated satellite-terrestrial multiantenna relay networks with multiuser scheduling," IEEE Trans. Aerosp. Electron. Syst., pp. 1-1, 2020.

[19] M. Abramowitz and I. A. Stegun, Handbook of Mathematical Functions with Formulas, Graphs, and Mathematical Tables, 10th ed. New York: Dover, 1972.

[20] A. P. Prudnikov, Y. A. Bryčkov, and O. I. Maričev, Integrals and Series: Special Functions, 3rd ed. New York: Gordon \& Breach Sci. Publ., 1992.

[21] V. S. Adamchik and O. I. Marichev, "The algorithm for calculating integrals of hypergeometric type functions and its realization in REDUCE system," in Proc. ISSAC. Tokyo, Japan: ACM, Aug. 1990, pp. 212-224. 